\title{
Exploring velocity limits in the thermonuclear supernova ejection scenario for hypervelocity stars and the origin of US 708 (Corrigendum)
}

P. Neunteufel ${ }^{1,2}$

\footnotetext{
1 Max Planck Institut für Astrophysik, Karl-Schwarzschild-Straße 1, 85748 Garching bei München, Germany e-mail: pneun@mpa-garching .mpg . de

${ }^{2}$ University of Leicester, University Road, LE1 7RH Leicester, Leicestershire, UK
}

A\&A 641, A52 (2020), https://doi.org/10.1051/0004-6361/202037792

Key words. binaries: close - supernovae: general - subdwarfs - white dwarfs - errata, addenda

Due to a typographical error in Table B.1, line three of the third column was reproduced in line four of the fourth column when that space should have been left blank. The corrected table is given as follows:
Table B.1. Parameters used in Eqs. (B.2)-(B.4).

\begin{tabular}{lcccc}
\hline \hline & $M_{\mathrm{b} / \mathrm{d} / \mathrm{h}}\left[M_{\mathrm{G}}\right]$ & $a_{\mathrm{d} / \mathrm{h}}[\mathrm{kpc}]$ & $b_{\mathrm{b} / \mathrm{d}}[\mathrm{kpc}]$ & $\Lambda[\mathrm{kpc}]$ \\
\hline Bulge $_{\mathrm{b}}$ & $409 \pm 63$ & & $0.23 \pm 0.03$ & \\
Disk $_{\mathrm{d}}$ & $2856_{-202}^{+376}$ & $4.22_{-0.99}^{+0.53}$ & $0.292_{-0.025}^{+0.020}$ & \\
Halo $_{\mathrm{h}}$ & $1018_{-603}^{+2793}$ & $2.562_{-1.419}^{+25.963}$ & & $200_{-82}^{+0}$ \\
\hline
\end{tabular}

